\title{
Protée
}

\section{Sémiotique et rhétorique musicales : la Fantaisie en ré mineur de Mozart}

\author{
Nicolas Meeùs et Jean-Pierre Bartoli
}

Volume 38, numéro 1, printemps 2010

Le Groupe $\mu$ entre rhétorique et sémiotique

URI : https://id.erudit.org/iderudit/039702ar

DOI : https://doi.org/10.7202/039702ar

Aller au sommaire du numéro

\section{Éditeur(s)}

Département des arts et lettres - Université du Québec à Chicoutimi

ISSN

0300-3523 (imprimé)

1708-2307 (numérique)

Découvrir la revue

Citer cet article

Meeùs, N. \& Bartoli, J.-P. (2010). Sémiotique et rhétorique musicales : la

Fantaisie en ré mineur de Mozart. Protée, 38(1), 55-64.

https://doi.org/10.7202/039702ar
Résumé de l'article

La Fantaisie KV 397 est examinée ici de deux points de vue différents mais complémentaires.La première approche est d'ordre analytique, inspirée par les travaux d'Heinrich Schenker (1868-1935) qui, dès 1930, avait anticipé dans le domaine de l'analyse musicale les concepts de la grammaire générative, décrivant l'oeuvre musicale comme le déploiement d'une structure profonde qu'il appelait Ursatz, « structure originelle ». Malgré la variété inhérente à l'écriture d'une Fantaisie, cette oeuvre manifeste une unité profonde dans le fait que chacune des parties dérive de la même structure originelle. La deuxième approche envisage la même oeuvre du point de vue d'une rhétorique générale inspirée du Groupe $\mu$, mais aussi des théories de Leonard Meyer (1918-2007). L'oeuvre est examinée ici en fonction de processus de création d'attentes, de ruptures d'isotopie, puis de résolution des attentes, de réévaluations proversives et rétroversives, ainsi que de la mise en oeuvre de catégories stylistiques établies (cantabile, tutti orchestral, antécédent/conséquent). Les deux analyses décrivent, chacune à sa manière, une intrigue proprement musicale, qui ne pourrait être traitée efficacement par une étude de type narratologique : les caractéristiques techniques de l'écriture mozartienne indiquent pourquoi l'oeuvre est demeurée inachevée.
Ce document est protégé par la loi sur le droit d'auteur. L'utilisation des services d’Érudit (y compris la reproduction) est assujettie à sa politique d'utilisation que vous pouvez consulter en ligne.

https://apropos.erudit.org/fr/usagers/politique-dutilisation/ 


\section{SÉMIOTIQUE ET RHÉTORIQUE MUSICALES: LA FANTAISIE EN RÉ MINEUR DE MOZART}

Nicolas Meeùs et Jean-Pierre Bartoli

Le problème de la sémiotique musicale, c'est celui du sens. Les membres de l'équipe de recherche «Patrimoines et Langages Musicaux» de l'Université ParisSorbonne sont d'opinion qu'il ne peut se résoudre uniquement par des tentatives de trouver à la musique un sens linguistique, un sens qui pourrait s'exprimer en langue quotidienne, en un mot de dire en langue ce que la musique raconte en musique. Si la musique est redevable d'une analyse sémiotique, c'est aussi pour d'autres raisons. C'est notamment que, comme le langage, elle est articulée selon un système économique, qui permet d'énoncer un nombre infini d'œuvres à partir d'un nombre réduit de notes; elle repose sur des stratégies discursives qui permettent de créer la cohérence ou la rupture, l'isotopie ou l'allotopie, la surprise, l'attente - réalisée ou (plus rarement) déçue. De tout cela naît un sens de la musique qui reste proprement indicible, mais qui, comme celui des langues quotidiennes, se construit par intégration d'unités d'un niveau donné dans des unités de niveau supérieur (Benveniste).

L'analyse sémiotique de la musique se ramène donc à l'analyse musicale elle-même qui a développé, de longue date, des techniques particulièrement sophistiquées. La difficulté de ces analyses tient à ce qu'elles supposent une compétence qui n'est pas universellement partagée, celle de la lecture de la musique. Nous rendant aux raisons de Sémir Badir lors de la préparation de ce dossier, nous ferons abstraction de cet obstacle, pour proposer une double analyse technique de la Fantaisie en ré mineur, KV 397. Nos références seront la théorie d'Heinrich Schenker ${ }^{1}$ (1868-1935), d'une part, et celle de Leonard B. Meyer ${ }^{2}$ (1918-2007), d'autre part. La rhétorique et la sémiotique du Groupe $\mu$ ont aussi constitué des sources d'inspiration constante.

La Fantaisie en ré mineur, dont la date de composition n'est pas connue et dont le manuscrit est perdu, n'a été publiée qu'en 1804 à Vienne, puis en 1806 à Leipzig. La première édition ne donne que 97 mesures, tandis que la seconde ajoute 10 mesures qui sont peut-être d'August Eberhard Müller (1767-1817)³. La version originale, interrompue à la mesure 97, n'est peut-être pas pour autant incomplète, mais elle se termine sans conclure, sur un accord de dominante, c'est-à-dire sur une tension non résolue et qui paraît appeler une continuation. S'agit-il d'un prélude à une œuvre qui manque (ou, du moins, qui n'a pas été identifiée comme devant faire suite à celle-ci), ou de la première partie d'une pièce dont la deuxième partie n'a pas été écrite? Quelles sont les raisons de cet inachèvement? Nous ne le saurons jamais, mais il est certain que 
cette situation poserait des problèmes insurmontables pour une interprétation de type narratologique: le dénouement de l'histoire n'est pas connu.

\section{ANALYSE SCHENKÉRIENNE par Nicolas Meeùs}

La théorie schenkérienne est une théorie de la musique tonale. Développée dans le premier tiers du XXe siècle, elle anticipe de près d'un demi-siècle la grammaire transformationnelle. Schenker conçoit l'œuvre tonale comme fondée sur une structure profonde, une «structure originelle» $\left(U_{\text {rsatz }}\right)^{4}$, dont il a donné plusieurs descriptions; au stade ultime de l'élaboration de la théorie, à partir de 1930, elle prend une forme canonique, constituée d'une ligne mélodique descendant vers la tonique (Urlinie, «ligne fondamentale»), appuyée sur une arpégiation I-V-I à la basse. Si la même structure profonde sert à toutes les ouvres tonales, chacune d'elles la réalise à sa manière: Schenker en avait fait sa devise, Semper idem, sed non eodem modo, «toujours la même chose, mais pas de la même manière». La description des œuvres, selon la théorie schenkérienne, passe donc par celle des processus génératifs par lesquels la structure originelle se transforme, à travers plusieurs paliers successifs, en la structure de surface, en la partition elle-même. Un aspect essentiel de la technique schenkérienne est la représentation graphique des niveaux de l'analyse, dont on trouvera de nombreux exemples ci-dessous.

Il faudrait pouvoir examiner ici le rapport entre la structure originelle schenkérienne et la structure profonde de la grammaire générative chomskyenne. Si les principes généraux sont de même nature, certaines différences sont importantes. D'abord, la grammaire générative, comme la plupart des grammaires des langues naturelles, concerne le niveau phrastique; la grammaire schenkérienne au contraire, comme la plupart des grammaires musicales, ne fait pas de distinction nette entre le niveau de la phrase et celui du discours: l'analyse ci-dessous le montrera à plusieurs reprises. Pour Schenker, la construction de l'œuvre à partir de sa structure originelle est un processus qui s'apparente à la fois à l'ornementation et à l'improvisation; on pense à l'analyse du discours en noyaux et catalyses chez Roland Barthes. Une autre différence essentielle est que Chomsky vise à décrire une capacité de langage universelle - donc peut-être innée -, tandis que Schenker, au contraire, pointe une pratique qu'il considère comme exceptionnelle, propriété exclusive du génie musical; sa définition du génie s'apparente d'ailleurs à une capacité de mettre en ouvre la grammaire qu'il décrit. Il ne sera pas possible d'en discuter plus longuement ici, mais il faut souligner brièvement que ce que Schenker décrit, c'est une manière assez répandue, mais certainement pas universelle, de pratiquer le langage tonal ${ }^{5}$; c'est en quelque sorte un style.

La Fantaisie en ré mineur de Mozart repose à plusieurs reprises et à plusieurs niveaux sur une structure originelle dont la forme la plus générale est celle de la figure 1, qui représente une première transformation de la structure fondamentale.

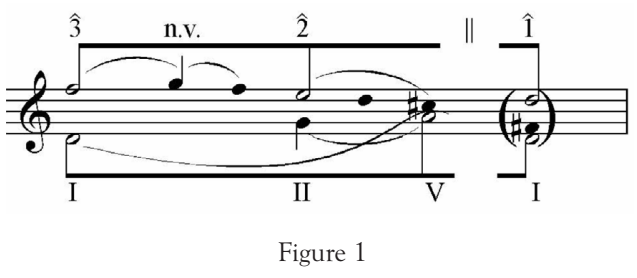

Les notes écrites en blanches sont celles de la structure fondamentale canonique, avec le chiffrage $\hat{3} \hat{2} \hat{1}$ ( $f a-$ mi-ré) pour la ligne fondamentale, IVI (ré-la-ré) pour la basse. L'accord final, entre parenthèses, est celui qui serait attendu pour conclure, mais qui manque dans la version de la première édition: la structure fondamentale est interrompue avant d'atteindre son but, comme l'indique ici le signe $\|$. Plusieurs ajouts personnalisent cette structure générale:

- La première note de la ligne fondamentale, $f a$ $(\widehat{3})$, est ornée par une broderie notée «n.v.» (note voisine).

- Le la de la basse (chiffré V) est préparé par la note sol, qui rend possible un accord "prédominant» du II e degré; la liaison à double courbe ${ }^{6}$ (qui représente une sinusoïde) souligne cette succession, toniqueprédominante-dominante (I-II-V).

- La ligne mélodique poursuit sa descente après avoir 
atteint le $m i(\hat{2})$, pour rejoindre par une note de passage, ré, la note sensible do\# qui appartient à une voix intérieure.

C'est cette structure fondamentale, sans son dernier accord, qui, sous une forme ou une autre, constitue la structure profonde de chacune des sections de la Fantaisie et assure l'unité de cette concaténation de parties sans grand lien organique apparent entre elles. Un niveau de développement ultérieur de la structure fondamentale servira d'une part à l'introduction, andante, et d'autre part au thème principal, adagio. Les modifications apportées sont les suivantes:

- La note voisine est amenée par un déploiement de l'accord du Ier degré, marqué dans la représentation graphique ci-dessous par la ligature oblique entre $f a$ $(\hat{3})$ et la.

- La descente vers le do\# se fait en passant par le mib plutôt que le mita, qui induit un accord de sixte napolitaine, chiffré bII.

- La montée sol-la, II-V, est complétée par un mouvement chromatique, sol-sol\#-la, qui permet de résoudre la sixte napolitaine sur le II ${ }^{e}$ degré "normal», chiffré ŁII.

La figure 2 ci-contre présente la structure fondamentale sous cette nouvelle forme.

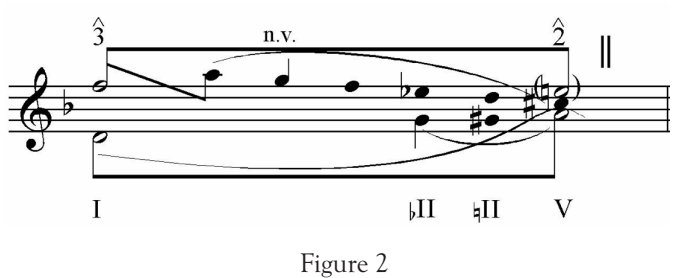

\section{INTRODUCTION, ANDANTE}

Pour l'introduction, la structure fondamentale subit encore une transformation, consistant surtout en l'ajout de notes de passage dans les intervalles disjoints du début; le saut de quarte ascendante à la basse, ré-sol, est transformé en une ligne de quinte descendante, ré-do-si-la-sol. La figure 3 ci-dessous indique la correspondance entre cette structure prolongée et le texte de Mozart ${ }^{7}$; quelques flèches de l'une à l'autre repèrent les correspondances principales.

\section{Adagio}

Le motif initial du thème principal de l'adagio, mesures $12-13$ ou 45-46, est un décalque presque exact de la structure fondamentale dans sa forme la plus simple, comme le montre la figure 4. Cette structure, couvrant à peine une mesure et demie, est enchâssée localement dans celle du thème lui-même, plus développé et qui compte huit ou neuf mesures.

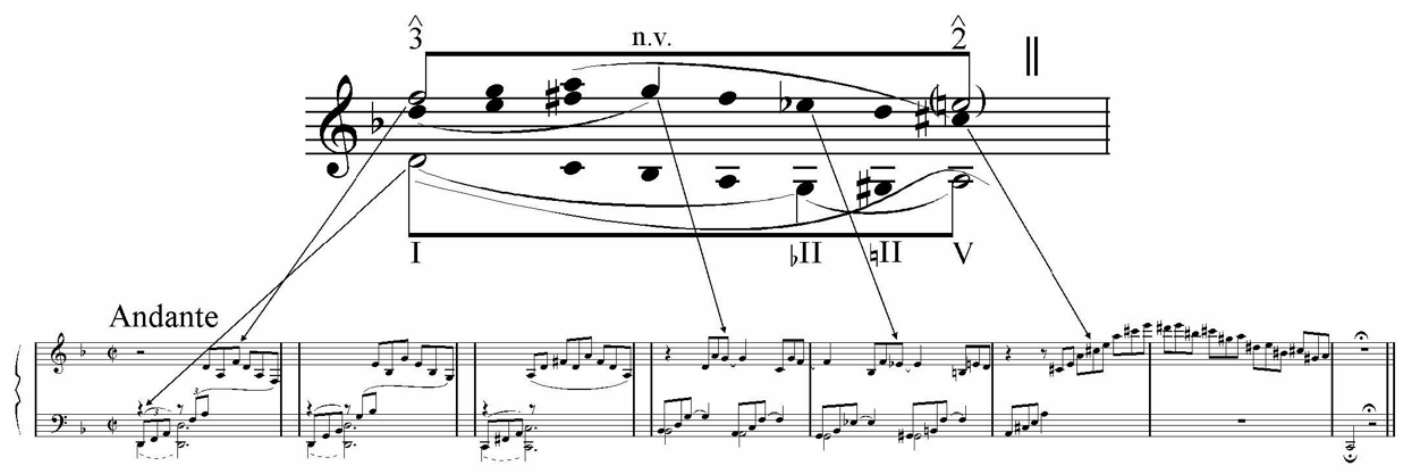

Figure 3
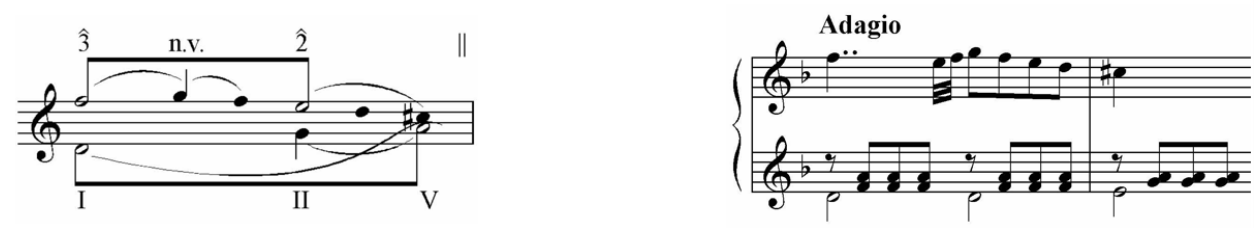

Figure 4 
Le thème principal est présenté deux fois, la première fois sous une forme un peu simplifiée au début de l'adagio, mesures 12-19, la deuxième fois, de la mesure 46 à la mesure 54 . C'est cette seconde présentation qui sera détaillée ici, parce qu'elle correspond à la structure profonde dans sa forme la plus caractéristique pour la Fantaisie, comprenant la sixte napolitaine, qui est élidée dans la première présentation. La figure 5 ci-dessous présente la structure fondamentale dans sa version déjà illustrée ci-dessus, figure 2, en regard d'une réduction de la partition des mesures 46-54.

On y voit le développement de chacun des éléments de la structure fondamentale: le déploiement de l'accord initial de ré mineur, avec son mouvement mélodie $f a-l a$ à la voix supérieure, se développe pour donner notamment la présentation du motif initial (mesures 45-46), déjà illustrée à la figure 4 ci-dessus; la reprise de celui-ci un degré plus haut ramène à la position initiale à la fin de la mesure 48, pour le saut fa-la, mesures 48-49. Le la est ensuite brodé par le sib, puis par un saut vers le ré aigu, mesure 50 , autorisant une descente de toute une octave, presque entièrement chromatique et passant par le mib de la sixte napolitaine, mesures 50-53.

Malgré ces figurations de surface, le lien à la structure profonde demeure étroit.

La partie centrale de l'adagio, mesures 20-44, est plus complexe et le lien à la structure profonde y est moins lisible, quoique toujours présent. La figure 6 ci-contre en donne une idée, qui ne sera cependant pas commentée dans le détail. On y lit d'abord,
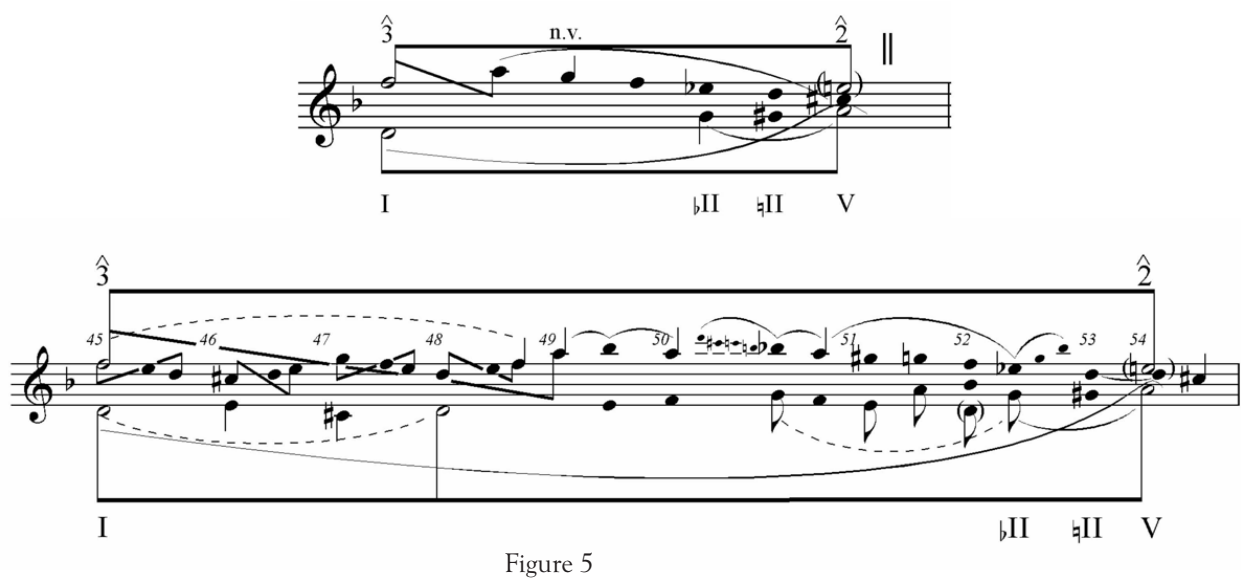

Figure 5
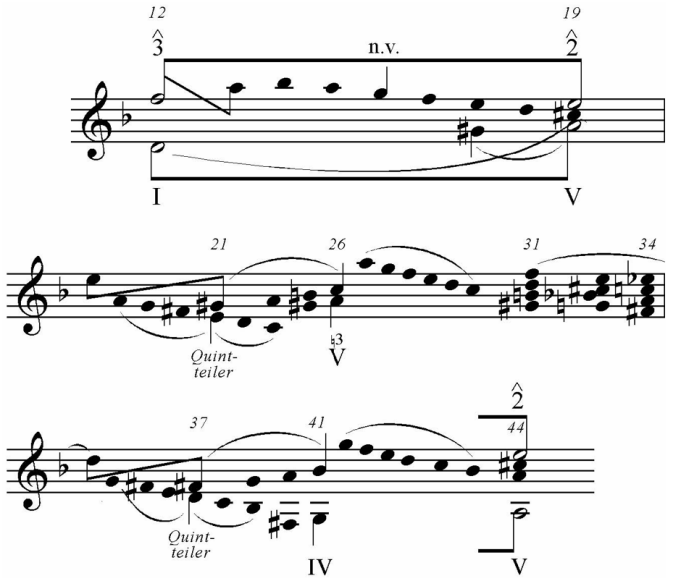

Figure 6

au-dessus, la version simplifiée de la présentation du thème, mesures 12-19, sans la sixte napolitaine. À la mesure 21, la dominante de la mesure 19 est ornée par sa propre quinte (dominante de la dominante), que Schenker appelle Quintteiler («diviseur à la quinte»); à la mesure 26, la dominante revient en mineur (comme il sied à une pièce dont la tonalité générale est mineure), avec un saut vers le la aigu qui reproduit celui de la structure fondamentale de la figure 2. Une descente mélodique s'opère ensuite vers le mib, qui apparaît non plus ici comme sixte napolitaine, mais comme nouveau «diviseur à la quinte», cette fois de la prédominante IV, qui ramène à l'accord de dominante terminant la partie centrale.

La figure 7 résume cette analyse: on y lit d'abord la réduction de l'andante introductif, tel qu'il a été présenté à la figure 3; puis la version brève du thème principal, sans le passage par la sixte napolitaine; la partie centrale de l'adagio n'est représentée que par sa dominante finale, parce qu'une analyse détaillée dépasserait le cadre de ce qui peut être fait ici (voir néanmoins la figure 6); enfin, le thème est présenté dans sa version complète, conformément à la figure 5 ci-contre. On y voit aussi l'exceptionnelle unité de cette œuvre, où tout est fait par ailleurs pour donner à l'auditeur le 


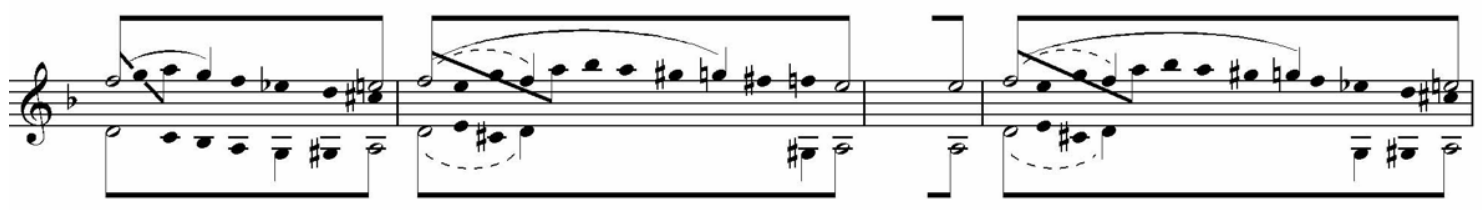

Figure 7

sentiment rhapsodique, improvisé et en apparence désordonné, qui caractérise le genre de la fantaisie. On ne manquera pas de noter aussi le caractère proprement «langagier», sémiotique, d'une telle unité organique, fondée sur une structure profonde qui se répète à plusieurs niveaux et qui assure l'économie de l'œuvre.

ANALYSE RHÉTORIQUE par Jean-Pierre Bartoli

La seconde analyse ici présentée se propose de revenir aux éléments de la surface de la partition et de l'observer à partir des catégories de la «néo-rhétorique structurale», telle qu'elle a pu être mise en ouvre à notre époque. L'un des mérites de celle-ci est en effet d'avoir forgé des outils à même de détacher la rhétorique de l'époque classique de son lieu d'origine - le domaine verbal - et d'avoir établi les fondements d'une «rhétorique générale» trans-sémiotique, comme l'attestent les textes désormais célèbres sur la rhétorique visuelle de Roland Barthes et plus encore ceux du Groupe $\mu$. Or, préférant le réexamen de l'esthétique baroque et l'étude des innombrables traités qui, aux XVII e et XVIII ${ }^{\mathrm{e}}$ siècles, reliaient la construction du discours musical aux figures de la rhétorique classique, la musicologie d'aujourd'hui n'a pas jugé bon de profiter du renouveau de la rhétorique contemporaine.

C'est au contraire dans ce dessein qu'il sera fait appel à la fois aux éléments de la description du fonctionnement rhétorique proposée par le Groupe $\mu$, auxquels s'adjoignent des outils puisés dans les écrits du musicologue Leonard B. Meyer, dont la teneur s'en approche singulièrement. Si ce dernier n'inscrit pas explicitement ses travaux dans le cadre rhétorique, mais plutôt dans celui de la psychologie des formes, il situe son approche de la musique classique en tentant de rendre compte des phénomènes d'attente induits chez l'auditeur par l'agencement des éléments musicaux produits par le compositeur. La notion, venue de la Gestalttheorie, d' «implication-réalisation" et les ruptures de ce principe au sein de données contextuelles bien précises s'approchent singulièrement de la description de la figure rhétorique telle qu'elle est décrite par le Groupe $\mu$, avec les notions sémiotiques d'isotopie et de rupture allotopique provoquant un phénomène d'impertinence.

Les signes d'analyse utilisés sont donc librement repris aux travaux du musicologue, tandis que les notions utilisées proviennent directement de ceux du Groupe $\mu$. Il est en effet question ici de repérer dans la Fantaisie de Mozart la production de figures à nature rhétorique qui articulent, sur le plan de la surface sonore, la perception de l'éloquence de l'œuvre et induisent une signification que l'on peut considérer comme proprement musicale dans la mesure où elle produit une sorte de narration. Cette dernière est pourtant dépourvue de sens linguistique, de sémantisme ou de référence à la réalité mondaine.

Il est au préalable nécessaire de livrer au lecteur quelques clés sur les signes qui seront à présent utilisés et placés sous ou sur les portées d'une partition, ou encore dans les schémas formels représentant le déroulement d'un énoncé musical, ou d'une ouvre entière. Tout d'abord, le déroulement homogène d'une séquence musicale cohérente est représenté sous la partition (ou sous un schéma qui la représente) par le signe (en forme de barquette) présenté dans la figure 8 .

Figure 8. Suite cohérente d'unités de signification.

Il désigne le déroulé homogène d'une séquence signifiante. Ici, il n'y a pas de rupture de la cohérence isotopique. C'est par exemple le cas dans le cadre de la musique tonale des configurations suivantes données à titre d'exemple: 
- cycle fonctionnel ( $c f)$, cadence ( $c p)$, cycle des quintes s'achevant sur une cadence...

- antécédent/conséquent, sentence (aab), forme binaire, exposition de forme sonate...

- modulation aux tons proches (dominante, relatif)... La rupture d'une telle séquence provoquée par le surgissement d'un élément allotope est indiquée par les signes présentés dans la figure 9.

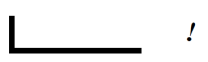

Figure 9. Production d'une figure rhétorique de rupture.

La rupture de la chaîne isotopique se produit par le surgissement d'une unité allotope qui produit une impertinence, laquelle est représentée par le point d'exclamation. L'élément de signification attendu peut être indiqué en italique. Ici, par exemple, dans la figure 10 qui représente le cas d'une figure harmonique, le chiffrage indiquant l'accord induit et espéré est en caractères italiques, tandis que les accords effectivement entendus sont en caractères romains.

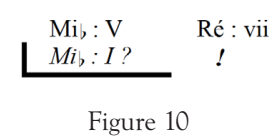

Le rétablissement de la cohérence isotopique peut se représenter par la figure 11 suivante:

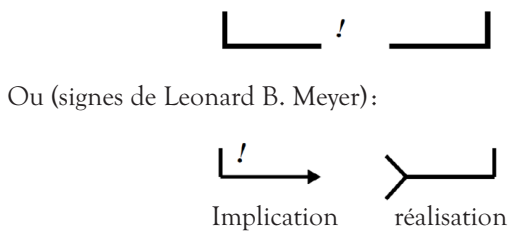

Figure 11. Rupture puis rétablissement de la cohérence de l'énoncé selon l'isotopie initiale.

Les phénomènes de réévaluation proversive et rétrospective, tels qu'ils sont décrits par le Groupe $\mu$, ainsi que d'autres situations singulières assez fréquentes en musique peuvent être représentés à leur tour selon la figure 12 .

Du point de vue de l'analyse de Nicolas Meeùs constituant le début du présent article, l'ensemble de la première partie de la Fantaisie de Mozart (andante

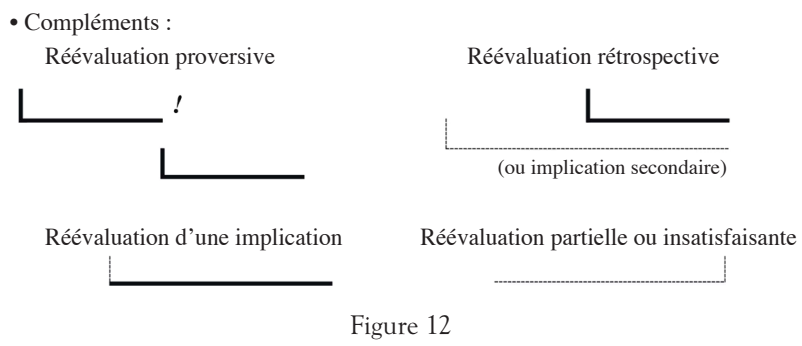

et adagio) possède sa cohérence parfaite dans la mesure où elle reproduit, à différents niveaux, un schéma générateur présenté dans l'andante initial. La conséquence de son analyse est que l'allegretto qui suit, laissé inachevé par Mozart, est en quelque sorte superflu. L'œuvre se conclut lors de la résolution de la cadence parfaite qui clôt la partie lente, et si Mozart s'arrête de composer quelques mesures plus tard, c'est que la structure génératrice de l'ouvrage a déjà été présentée et qu'il ne reste plus rien d'essentiel à inventer. L'analyse que l'on va présenter ici apporte, sur le plan de la surface thématique, la confirmation de l'hypothèse de Nicolas Meeùs.

Une fois présentées les onze mesures initiales de tempo andante, lesquelles déploient en arpèges une progression de la tonique à la dominante, apparaît clairement un début de thème de tempo adagio écrit dans le style cantabile - on croirait entendre le début d'un air de soprano. Ces mesures 12 à 19 possèdent toutes les caractéristiques d'un antécédent ${ }^{8}$. Ponctué par une demi-cadence, celui-ci est en effet composé de deux sections de quatre mesures chacune, et, selon les usages, il fait attendre l'apparition immédiate d'un conséquent qui reprendrait, de façon conclusive, les deux sections en s'achevant, cette fois, sur une cadence parfaite. La figure 13 présente l'antécédent tel qu'il est écrit par Mozart (et son analyse par signes commentée plus bas) et la figure 14, le schéma de la structure attendue.

C'est alors que Mozart provoque une franche rupture d'écriture en faisant surgir, en lieu et place du conséquent, une formule chromatique scandée forte dans le grave du clavier comme l'intervention dramatique d'un tutti d'orchestre (points d'exclamation dans la figure 15). L'antécédent se retrouve seul et le conséquent prévisible ne survient pas. 


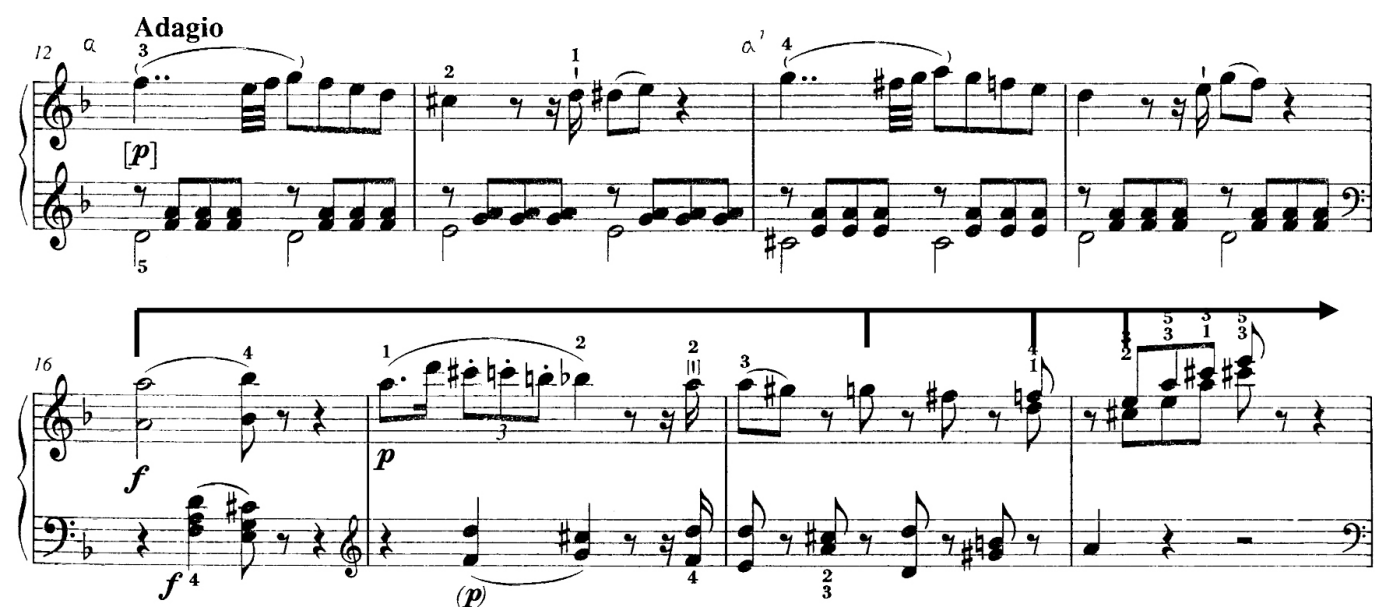

Figure 13. Antécédent du thème.

Du point de vue mélodique, cette attente se traduit dans la seconde moitié du conséquent par une descente depuis le la aigu jusqu'au mi laissant espérer un ré qui ne vient pas (voir la flèche dans la figure 13 et la figure 14). La résolution sur le ré et l'accord de tonique dans un conséquent résolutif va-t-elle finalement intervenir et quand? Tel est le nœud de la courte tragédie dans laquelle Mozart entraîne l'auditeur. Le tutti d'orchestre laisse place à une partie en forme de récitatif agité qui doit être jouée en accelerando. L'image du théâtre s'accroît: il semble ainsi que la soprano réagit au tutti. Reviennent alors les premières mesures du thème. Estce le conséquent? Non: joué non pas à la tonique, mais en la mineur, le thème est bientôt dévié et la tentative de résolution tourne court. Un trait virtuose vient à son tour interrompre le processus et l'on se retrouve au point de départ du drame. On réentend le tutti puis le récitatif. Mais cette fois, passant par la sous-dominante, le parcours tonal se dirige vers la tonique. La reprise du trait ramène le thème: le conséquent attendu intervient alors et la descente du la vers le ré est enfin complète (quoique retardée par des arpèges). Ainsi, avec la résolution de l'attente engendrée, s'achève ce mini-drame instrumental.

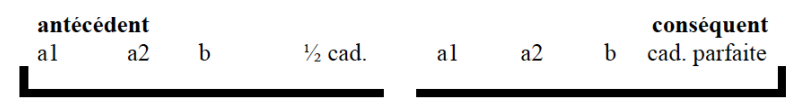

Figure 14

Structure de référence du thème de la Fantaisie en ré mineur, K. 397, de Mozart. Isotopie: antécédent/conséquent de la formule de type "période».
Le schéma ${ }^{9}$ de la figure 16 représente l'intrigue fondée sur la perturbation de l'isotopie <antécédent/ conséquent $>$. Il faut noter que les éléments qui dérangent le déroulement prévu, ou ceux qui sont consécutifs à ces ruptures, relèvent non pas de la catégorie des thèmes, mais de gestes spectaculaires de nature dramatique: un tutti semblant commenter quelque arrivée de statue du Commandeur, un récitatif haletant qui rappelle la figure rhétorique de la suspiratio, des traits virtuoses comme s'ils représentaient une course éperdue. Un seul thème est présent dans cette fantaisie et il faudrait même dire, pour être plus exact, que ce mouvement tout entier se résume à une difficile gestation: un antécédent cherche son conséquent.

La figure 17 rapproche les mesures 12 à 20 des mesures 45 à 55 pour montrer qu'elles auraient pu s'agencer en continuité. Entre les mesures 20 et 45 se multiplient les effets «impertinents» qui entretiennent l'attente de l'auditeur. Par une analyse différente de celle de Nicolas Meeùs, il se confirme que l'intrigue de la pièce se termine à l'arrivée de l'allegretto qui marque l'achèvement du conséquent enfin entendu en entier. Si celui-ci se prolongeait trop, il ressemblerait à un trop long lieto finale d'opera seria. Ce à quoi Mozart s'est refusé en ayant remis à plus tard la rédaction d'une conclusion plus concise que celle primitivement imaginée, et l'on peut féliciter Müller de ne pas avoir développé cette section qui ne comporte effectivement plus aucun enjeu. 

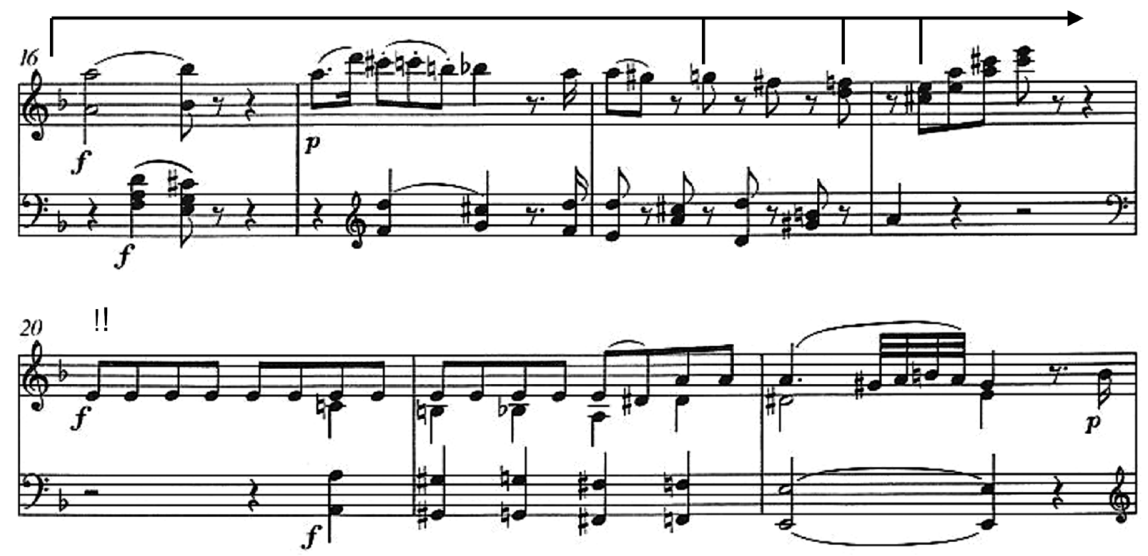

Figure 15

Mozart, Fantaisie en ré mineur K. 397 (385 g)

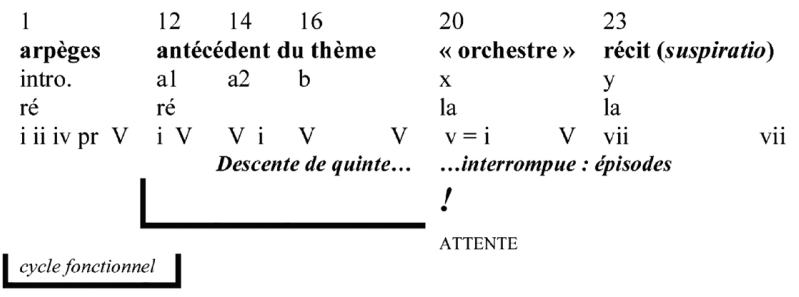

$29 \quad 31$

début conséquent

$\begin{array}{ll}\text { al a2' } & \text { a } \\ \text { la }\end{array}$

i vii vii

mauvaise tonalité : parcours dévié..

! !

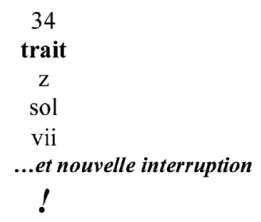

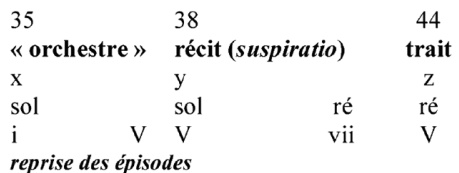

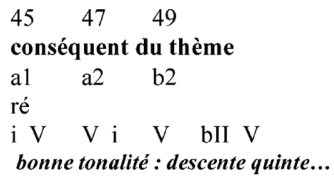

4

$\mathrm{V}$

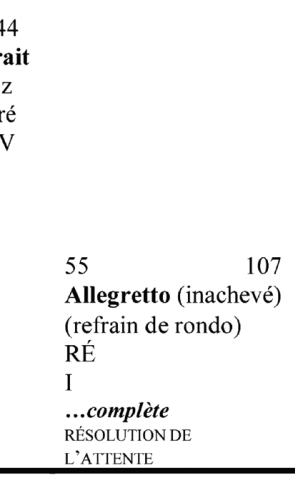

Figure 16. Mozart, Fantaisie en ré mineur, K. 397, schéma paradigmatique. 
ANTÉCÉDENT
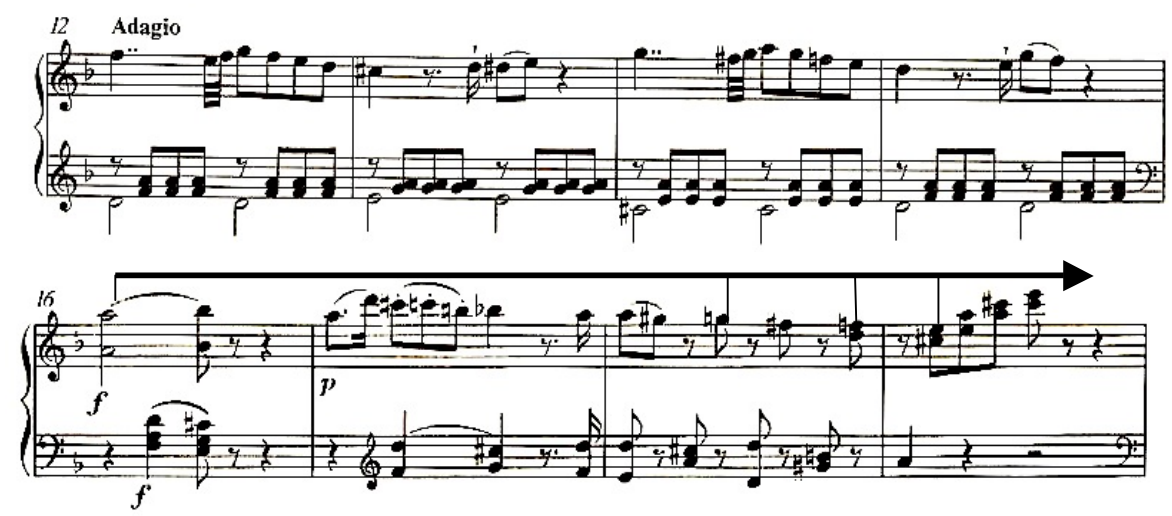

CONSÉQUENT
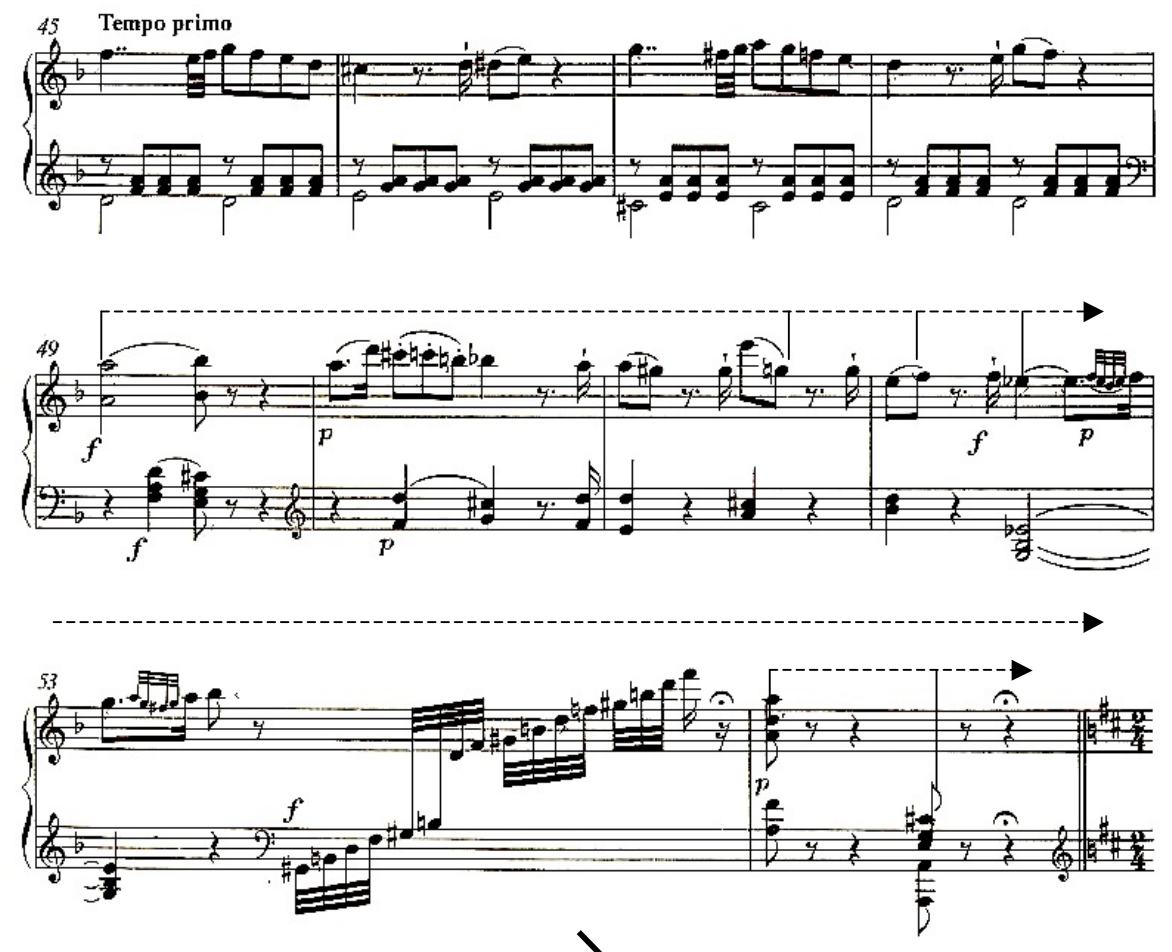

FIN DU CONSÉQUENT (fin de l'Allegretto)

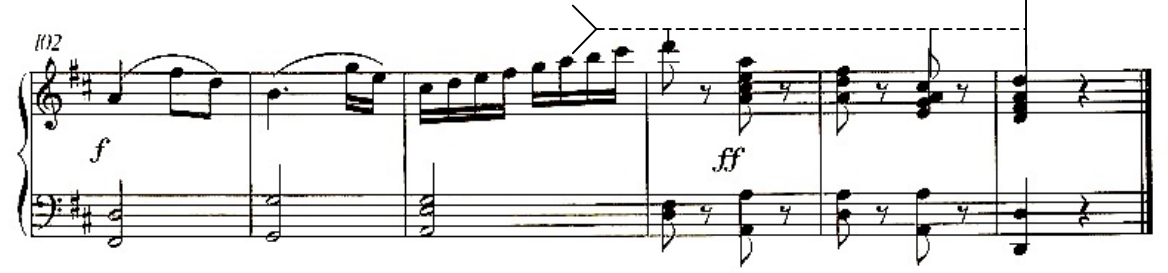

Figure 17 
Les deux analyses ci-dessus n'ont évidemment pas la prétention d'épuiser l'analyse de l'œuvre du point de vue musicologique. Leur ambition est seulement de montrer qu'il est possible et légitime d'aborder une pièce comme cette Fantaisie de Mozart - et, plus généralement, le langage musical lui-même - d'un point de vue sémiotique et rhétorique; ou, en d'autres termes, une sémiotique et une rhétorique proprement musicales pourraient n'être ni utopiques, ni pédantes, ni abstruses. La question de la possibilité d'étendre la sémiotique au langage musical avait déjà été posée par Hjelmslev, lorsqu'il s'était interrogé sur un éventuel isomorphisme des plans de l'expression et du contenu musical. Cette question est demeurée ouverte, polluée à notre avis par une conception par trop référentielle du sens. Traçant, mieux que quiconque, le cadre d'une sémiotique et d'une rhétorique générales, le Groupe $\mu$ a créé un espace et des outils qui rendent possible le développement d'une sémiotique et d'une rhétorique musicales véritables, non plus comme des décalques ou des extensions insuffisamment justifiées des disciplines linguistiques, mais comme partie prenante autonome des disciplines générales. Si le Groupe $\mu$ ne s'est pas aventuré explicitement dans le domaine musical, il paraît évident aux musicologues que nous sommes qu'il n'en a jamais été très éloigné.

\section{NOTES}

1. Le seul ouvrage de Schenker traduit en français est L'Écriture libre, ouvrage posthume de 1935 (trad. de N. Meeùs, Liège, Mardaga, 1993). Pour une liste des écrits de Schenker, voir N. Meeùs (1993).

2. Notamment Emotion and Meaning in Music; Explaining Music; Style and Music.

3. Sur cette question, voir P. Hirsch (1944); R. Steglich (1954); Plath (1973).

4. Le terme Urlinie, «ligne originelle», apparait pour la première fois en 1920, dans l'analyse publiée par Schenker de la Sonate op. 101 de Beethoven; le terme Ursatz apparait en 1923. Mais on montrerait aisément que les notions que ces termes recouvrent existent implicitement dans des analyses antérieures. L'usage moderne est de traduire Urlinie et Ursatz par «ligne fondamentale» et «structure fondamentale", mais il est aujourd'hui discuté. Je remercie Anne-Marie Houdebine d'avoir soulevé ce point au moment du débat qui a suivi la présentation orale de cette analyse: elle m'a convaincu de modifier mon usage, de préférer désormais «ligne originelle» et «structure originelle " et de réserver les expressions «ligne fondamentale» et "structure fondamentale" à la désignation particulière de la structure profonde dans sa forme canonique, décrite par Schenker à partir de 1930.

5. Schenker lui-même en fait l'argument d'un jugement de valeur: les œuvres qui ne se conforment pas à sa grammaire sont sans génie. Il serait imprudent de le suivre dans cette argumentation. 6. Le terme "prédominant" ne fait pas partie du vocabulaire schenkérien, mais Schenker paraît avoir pensé qu'une structure fondamentale complète devait comporter un accord de ce type: c'est pourquoi il a imaginé la liaison à double courbe signalée ici.

7. Pour la concision, les mesures répétées (1-2, 3-4 et 5-6) ne sont données qu'une fois.

8. Antécédent d'un thème de type "période», pour reprendre la typologie désormais classique de Schoenberg ou W. E. Caplin (1998). 9. Il se lit de gauche à droite (axe syntaxique) et de haut en bas (axe paradigmatique). Sont alignées verticalement les séquences paradigmatiquement liées.

\section{RÉFÉRENCES BIBLIOGRAPHIQUES}

BARTHES, R. [1981]: «Introduction à l'analyse structurale des récits », L'Analyse structurale du récit, Paris, Seuil, 7-33.

CAPLIN, W.E. [1998]: Classical Form, a Theory of Formal Functions for the Instrumental Music of Haydn, Mozart, and Beethoven, Oxford, New York, Oxford University Press.

CHOMSKY, N. [1969]: Structures syntaxiques, Paris, Seuil; [1972]: Le Langage et la Pensée, Paris, Payot.

Groupe $\mu$ [(1970) 1982]: Rhétorique générale, Paris, Seuil, coll. «Points Essais ";

[(1977) 1990]: Rhétorique de la poésie. Lecture linéaire, lecture tabulaire, Paris, Seuil, coll. «Points Essais»; Seuil.

HiRsCH, P. [1944]: «A Mozart Problem”, Music E Letters XXV, 209-212. KLinkenberg, J.-M. [2000]: Précis de sémiotique générale, Paris, Seuil, coll. «Points Essais».

MEEÙs, N. [1993]: Heinrich Schenker. Une introduction, Liège, Mardaga. MeYER, L. B. [1956]: Emotion and Meaning in Music, Chicago, University of Chicago Press;

_ [1973]: Explaining Music, Berkeley, University of California Press;

_ [1989]: Style and Music, Philadelphia, University of Pennsylvania Press.

Plath [1973]: «Zur Echtheidsfrage bei Mozart », Mozart Jahrbuch 1971/72, Salzburg, 19-36.

Steglich, R. [1954]: «Über das melodische Motiv in der Musik Mozarts. Eine Analyse der d-moll-Phantasie für Klavier ", Mozart Jahrbuch 1953, Salzburg, 128-142. 